\title{
MAPS BETWEEN ORBIFOLDS
}

\author{
MASAYUKI YAMASAKI
}

(Communicated by Frederick R. Cohen)

\begin{abstract}
Elementary homotopy theory on maps between orbifolds is discussed. For example, it is shown that, given a homomorphism $\varphi$ between orbifold fundamental groups of certain orbifolds, there exists a map (unique up to homotopy) between the orbifolds which induces $\varphi$. We also study the properties of orbifolds preserved by homotopy-equivalences.
\end{abstract}

\section{INTRODUCTION}

In $[10,11]$ Takeuchi studied orbifolds and maps between them, and showed that orbifolds with isomorphic orbifold fundamental groups are isomorphic in certain occasions. Maps between low dimensional orbifolds were the main concern. The aim of this paper is to discuss elementary homotopy theory on maps between orbifolds in a more general setting.

In $\S 2$, after a review on orbifolds, we introduce the notion of "orbi-maps" that was defined by Takeuchi. Actually he has several different versions of orbimaps, and the one given here is what the author thinks best. Roughly speaking a map is an orbi-map if it is covered by an equivariant map of the universal covering orbifolds. The main result of this section is a homotopy classification of orbi-maps with an aspherical target orbifold $(2.4,2.5)$.

In $\S 3$ we restrict ourselves to smaller classes of maps, i.e., tame maps and OR-maps. The key observation is that a tame map between good orbifolds which induces a monomorphism on orbifold fundamental groups induces a monomorphism between local groups $(3.5,3.6)$. If the map is an OR-map in addition, then the corresponding local groups must be isomorphic (as groups). This forces the map to preserve stratification (3.8).

In $\S 4$, we study "homotopy equivalences" between orbifolds. If two orbifolds are OR-homotopy equivalent, then it will be shown that one is good if and only if the other is good (4.2). We also show that OR-homotopy equivalences between

Received by the editors March 7, 1989.

1980 Mathematics Subject Classification (1985 Revision). Primary 57N80; Secondary 57M12.

Key words and phrases. Orbifold, orbi-map, tame map, OR-map.

The author was partially supported by MESC Grant-in-Aid for Scientific Research. 
2-dimensional closed orbifolds is OR-homotopic to an isomorphism (4.4). A similar result is also proved in [10].

\section{ORBI-MAPS}

An $n$-dimensional orbifold (possibly with boundary) is a Hausdorff paracompact space which is locally homeomorphic to the quotient space of $\mathbf{R}^{n}$ (or $\mathbf{R}_{+}^{n}=\mathbf{R}^{n-1} \times[0, \infty)$ ) by a finite orthogonal transformation group. (See [12, 9, 2], ... for the precise definition.) Thus each point $x$ of a connected orbifold $X$ is equipped with the following data: an open neighborhood $U_{x}$ of $x$ in $X$ (a coordinate neighborhood), a subgroup $\Gamma(x)$ of $O(n)$ (the local group at $x$ ), and a homeomorphism $\psi_{x}: \mathbf{R}^{n} / \Gamma(x) \rightarrow U_{x}\left(\right.$ or $\left.\mathbf{R}_{+}^{n} / \Gamma(x) \rightarrow U_{x}\right)$ sending the orbit of the origin to $x . X$ has a stratification by local group type. $\Sigma X$ denotes the singular locus of $X$; i.e., $\Sigma X$ is the set of points in $X$ at which the local group is nontrivial. $X$ is good if the singular locus of its orbifold universal cover $\widetilde{X}$ is empty [12]. In this paper all the orbifolds are assumed to be connected.

Suppose $X$ is a branchfold in the sense of Kato [6], i.e., an orbifold without codimension 1 strata. Then $X_{0}=X-\Sigma X$ is the unique top dimensional stratum. Let $p_{X}: \tilde{X} \rightarrow X$ denote the orbifold universal cover. It is a regular branched cover with branch locus $\Sigma X$. The deck transformation group is called the orbifold fundamental group and is denoted $\pi_{1}^{\text {orb }}(X)$. When restricted to $X_{0}, p_{X}$ is the ordinary covering associated with the subgroup $N_{X}$ of $\pi_{1}\left(X_{0}\right)$ normally generated by $\mu_{i}^{b_{1}}$ 's, where $\mu_{i}$ is the meridian loop in $X_{0}$ about a codimension 2 stratum $S_{i}$ and $b_{i}$ is the order of the local group along $S_{i}$; therefore, we can identify $\pi_{1}^{\text {orb }}(X)$ with $\pi_{1}\left(X_{0}\right) / N_{X}$ by choosing a base point in $p_{X}^{-1}\left(X_{0}\right)$. Let $q_{X}: \pi_{1}\left(X_{0}\right) \rightarrow \pi_{1}\left(X_{0}\right) / N_{X} \cong \pi_{1}^{\text {orb }}(X)$ denote the natural projection.

If we have another branchfold, say $Y$, then $\Sigma Y, Y_{0}, p_{Y}: \widetilde{Y} \rightarrow Y$, etc. are defined in the same way.

Let $X$ and $Y$ be connected branchfolds, and $f: X \rightarrow Y$ be a continuous map. The restriction $f \mid X_{0}-f^{-1}(\Sigma Y): X_{0}-f^{-1}(\Sigma Y) \rightarrow Y_{0}$ of $f$ will be denoted $f_{0}$ (assuming $X_{0}-f^{-1}(\Sigma Y)$ is nonempty), and the inclusion map of $X_{0}-f^{-1}(\Sigma Y)$ in $X_{0}$ is denoted $i$. We choose a base point of $X_{0}-f^{-1}(\Sigma Y)$ and we use it (resp. its image in $Y_{0}$ ) as the base point of $X_{0}$ (resp. $Y_{0}$ ). Let $K_{f}$ denote the kernel of $q_{X} \circ i_{*}: \pi_{1}\left(X_{0}-f^{-1}(\Sigma Y)\right) \rightarrow \pi_{1}^{\text {orb }}(X)$, then a component of $p_{X}^{-1}\left(X_{0}-f^{-1}(\Sigma Y)\right)$ is a covering of $X_{0}-f^{-1}(\Sigma Y)$ with deck transformation group $\pi_{1}\left(X_{0}-f^{-1}(\Sigma Y)\right) / K_{f}$. When the composition $q_{X} \circ i_{*}$ is surjective, this group is isomorphic to $\pi_{1}^{\text {orb }}(X)$ and $p_{X}^{-1}\left(X_{0}-f^{-1}(\Sigma Y)\right)$ is connected.

Definition 2.1. A map $f: X \rightarrow Y$ is a pre-orbi-map if $q_{X} \circ i_{*}$ is surjective and $\left(f_{0}\right)_{*}\left(K_{f}\right) \subset N_{Y}$. 
If $f$ is a pre-orbi-map, we have the following commutative diagram:

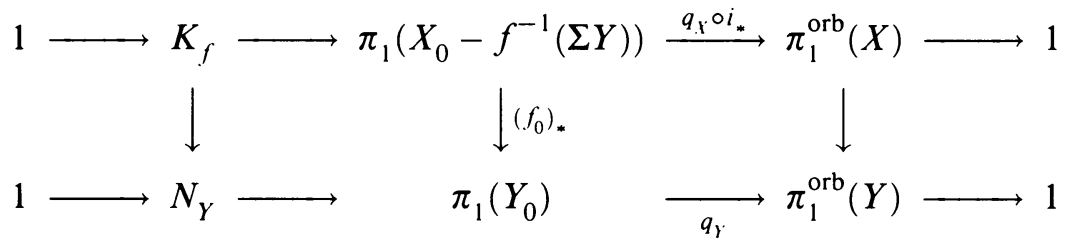

where the horizontal sequences are exact. The homomorphism $\pi_{1}^{\text {orb }}(X) \rightarrow$ $\pi_{1}^{\text {orb }}(Y)$ induced by $\left(f_{0}\right)_{*}$ is denoted $f_{*}$, and is called the homomorphism induced by $f$.

Note that, if $f$ is a pre-orbi-map, $f_{0}: X_{0}-f^{-1}(\Sigma Y) \rightarrow Y_{0}$ is covered by an $f_{*}$-equivariant map $\tilde{f}_{0}: p_{X}^{-1}\left(X_{0}-f^{-1}(\Sigma Y)\right) \rightarrow p_{Y}^{-1}\left(Y_{0}\right)$ which is unique up to the action of $\pi_{1}^{\text {orb }}(Y)$. The following definition is due to Takeuchi.

Definition 2.2. A pre-orbi-map $f: X \rightarrow Y$ is called an orbi-map if there exists an $f_{*}$-equivariant map $\tilde{f}: \widetilde{X} \rightarrow \widetilde{Y}$ which covers $f$ (i.e., $p_{Y} \circ \tilde{f}=f \circ p_{X}$ ). $\tilde{f}$ is called the universal map of $f$.

Suppose $\varphi: \pi_{1}^{\text {orb }}(X) \rightarrow \pi_{1}^{\text {orb }}(Y)$ is a homomorphism. A $\varphi$-equivariant map $\tilde{f}: \tilde{X} \rightarrow \tilde{Y}$ induces a map $f: X \rightarrow Y$; it is an orbi-map if and only if the composition $\pi_{1}\left(X_{0}-f^{-1}(\Sigma Y)\right) \rightarrow \pi_{1}\left(X_{0}\right) \rightarrow \pi_{1}^{\text {orb }}(X)$ is onto. If $f$ is an orbi-map, then $f_{*}=\varphi$.

Definition 2.3. An orbi-homotopy is a homotopy which is an orbi-map. If there is an orbi-homotopy between two orbi-maps, they are said to be orbi-homotopic. Obviously, if two orbi-maps are orbi-homotopic fixing the base point, then they induce the same homomorphism between the orbifold fundamental groups.

Takeuchi's proof of [11, Theorem 4.2] can be adapted to prove the following existence and uniqueness theorems. See the definition below for the terminology.

Theorem 2.4. Suppose $Y$ is an aspherical branchfold. Then for any connected branchfold $X$ and for any homomorphism $\varphi: \pi_{1}^{\mathrm{orb}}(X) \rightarrow \pi_{1}^{\mathrm{orb}}(Y)$, there exists an orbi-map $f: X \rightarrow Y$ with $f_{*}=\varphi$.

Theorem 2.5. Suppose $Y$ is a good aspherical branchfold. Then two base-pointpreserving orbi-maps $f, g: X \rightarrow Y$ which induce the same homomorphism $\varphi: \pi_{1}^{\mathrm{orb}}(X) \rightarrow \pi_{1}^{\mathrm{orb}}(Y)$ are orbi-homotopic fixing the base point.

Definition 2.6. A connected orbifold $X$ is called aspherical if the fixed set $\widetilde{X}^{H}$ is contractible for every finite subgroup $H$ of $\pi_{1}^{\text {orb }}(X)$. (In particular, $\widetilde{X}^{H}$ must be nonempty.)

Examples 2.7. (1) If $M$ is a simply-connected complete Riemannian manifold with nonpositive sectional curvature and $G$ is a discrete subgroup of the group of isometries of $M$, then the orbit space $M / G$ is an aspherical orbifold with orbifold fundamental group $G$. 
(2) A three-dimensional orbifold whose orbifold universal cover is homeomorphic to $\mathbf{R}^{3}$ and whose orbifold fundamental group has no subgroup isomorphic to $A_{5}$ is an aspherical orbifold [8].

(3) Let $(\widetilde{M}, \Gamma)$ be a crystallographic manifold in the sense of ConnollyKozniewski [1]. Then $\widetilde{M} / \Gamma$ is an aspherical (topological) orbifold.

Theorems 2.5 and 2.6 are proved inductively on strata of $X$. The local group $\Gamma(x)$ and the orbifold fundamental group $G=\pi_{1}^{\text {orb }}(X)$ of a branchfold $X$ are related in the following way. Let $U_{x}$ be a coordinate neighborhood of $x$ in $X$. Let us identify $U_{x}$ with $\mathbf{R}^{n} / \Gamma(x)$ (or $\mathbf{R}_{+}^{n} / \Gamma(x)$ in case $x$ is a boundary point). The inclusion map $i: U_{x} \rightarrow X$ is an orbi-map and induces a homomorphism

$$
i_{*}: \Gamma(x)=\pi_{1}^{\text {orb }}\left(U_{x}\right) \rightarrow \pi_{1}^{\text {orb }}(X)=G
$$

(In fact it is an "OR-map." See $\S 3$.) Let $\tilde{l}$ denote the universal map for $i$. Then it is easily verified that the image $i_{*}(\Gamma(x))$ is the isotropy subgroup $G_{i(O)}$ of $G$ at $\tilde{l}(O)$, where $O$ is the origin of $\mathbf{R}^{n}$. If $y$ is a point of the closure of the stratum containing $x$, then $i_{*}$ factors through $\Gamma(y)$. Therefore the stratification of $\tilde{X}$ induced from that of $X$ respects the isotropy subgroups. In general $i_{*}$ is not injective; $i_{*}$ is injective for every $x \in X$ if and only if $X$ is $\operatorname{good}[2$, p.186; 6, p.153].

Proof of Theorem 2.4. Triangulate $X$ so that $\Sigma X$ is a subcomplex of $X$. Let $X_{0}=S_{0}, S_{1}, \ldots, S_{l}$ be the set of strata of $X$ in order of nondecreasing codimension. $S_{i}^{*}$ (resp. $N\left(S_{i}^{*}\right)$ ) will denote the portion of $S_{i}$ (resp. the regular neighborhood of $S_{i}$ ) outside of the interior of the union of regular neighborhoods of lower dimensional strata, where $i=1, \ldots, l$.

First choose any map from the 1-skeleton of $S_{0}^{*}$ to $Y_{0}$ which induces $\varphi$. We can extend this map to obtain a map $S_{0}^{*}=N\left(S_{0}^{*}\right) \rightarrow Y$. Actually we construct a $\varphi$-equivariant map $p_{X}^{-1}\left(S_{0}^{*}\right) \rightarrow \widetilde{Y}$ inductively on skelta. This is possible because $\widetilde{Y}$ is contractible.

Assume inductively that we have constructed a $\varphi$-equivariant map $p_{X}^{-1}\left(N\left(S_{0}^{*}\right)\right.$ $\left.\cup \cdots \cup N\left(S_{i-1}^{*}\right)\right) \rightarrow \tilde{Y}$. Pick up a connected component $C$ of $p_{X}^{-1}\left(S_{i}^{*}\right)$ and let $H$ be the isotropy subgroup corresponding to it. The fixed set $\widetilde{Y}^{H}$ is nonempty, so we can map $C$ into $\tilde{Y}^{H}$, say by a constant map, and can extend it $\varphi$-equivariantly over $p_{X}^{-1}\left(S_{i}^{*}\right) . N\left(S_{i}^{*}\right)$ has a locally trivial bundle structure $\pi: N\left(S_{i}^{*}\right) \rightarrow S_{i}^{*}$. Fix a triangulation of $S_{i}^{*}$, and assume inductively that we have already extended the map $\varphi$-equivariantly over $p_{X}^{-1}\left(\pi^{-1}\left(\left(S_{i}^{*}\right)^{(j-1)}\right)\right)$, where $\left(S_{i}^{*}\right)^{(j-1)}$ denotes the $(j-1)$-skeleton of $S_{i}^{*}$. Let $\Delta$ be a simplex of $S_{i}^{*}$. The fiber of $\pi$ over a point $x \in \Delta$ can be identified with a cone of the form $\{x\} * K$. Triangulate $K$ and give $\pi^{-1}(\Delta)$ the product CW structure of $\Delta$ and the cone $\{x\} * K$. We can arrange so that, for any stratum $S$ of $X, \bar{S} \cap \pi^{-1}(\Delta)$ is a subcomplex of $\pi^{-1}(\Delta)$. Here $\bar{S}$ denotes the closure of $S$. 
Suppose inductively that we have extended the map $\varphi$-equivariantly over $p_{X}^{-1}\left(\Delta \times\left(\{x\} * K^{(k-1)}\right)\right)$, where $K^{(k-1)}$ denotes the $(k-1)$-skeleton of $K$. Let $\sigma$ be a simplex of $K$ and let $\tau$ be a component of $p_{X}^{-1}(\Delta \times(\{x\} * \sigma))$. It is a cell. Let $F$ be the isotropy subgroup at an interior point of $\tau$. By assumption $\partial \tau$ is mapped into $\widetilde{Y}^{\varphi(F)}$, which is contractible. Therefore, the map $\partial \tau \rightarrow \widetilde{Y}^{\varphi(F)}$ extends to a map $\tau \rightarrow \widetilde{Y}^{\varphi(F)}$. We can further extend this over $p_{X}^{-1}(\Delta \times(\{x\} * \sigma))$ $\varphi$-equivariantly. Thus we can construct a $\varphi$-equivariant map $p_{X}^{-1}\left(\pi^{-1}(\Delta)\right) \rightarrow \widetilde{Y}$ by induction on skelta of $K$, and this in turn gives the inductive step to extend the original map $\varphi$-equivariantly over $p_{X}^{-1}\left(S_{i}^{*}\right)$. Now, by induction on $i$, we have the desired map.

To prove 2.5, we introduce the notion of 0-transverse orbi-maps.

Definition 2.8. Let $X$ and $Y$ be branchfolds. Assume $Y$ is good. An orbi-map $f: X \rightarrow Y$ is 0 -transverse if the restriction $\widetilde{f} \mid p_{X}^{-1}\left(X_{0}\right): p_{X}^{-1}\left(X_{0}\right) \rightarrow \widetilde{Y}$ of the universal map is transeverse to $p_{Y}^{-1}(T) \subset \widetilde{Y}$ for each stratum $T$ of $Y$.

Proposition 2.9. Let $Y$ be a good branchfold. Any orbi-map $f: X \rightarrow Y$ from a branchfold is orbi-homotopic to a 0-transverse orbi-map.

Proof. Suppose $X$ is an $n$-dimensional orbifold. Let $B$ and $B^{\prime}$ be a pair of small $n$-dimensional balls in $X_{0}$ with $B^{\prime}$ contained in the interior of $B$. Let $B_{1}$ be a component of $p_{X}^{-1}(B)$ and $B_{1}^{\prime}$ be a component of $p_{X}^{-1}\left(B^{\prime}\right)$ contained in $B_{1}$. By an inductive application of the standard transversality argument, we can modify $\tilde{f} \mid B_{1}$ rel $\partial B_{1}$ so that $\tilde{f} \mid B_{1}^{\prime}$ is transverse to the preimage of every stratum of $Y$ in $\tilde{Y}$. We can make the modification sufficiently small so that $\tilde{f}$ stays transverse at points where it is already transverse. Extend the modification $f_{*}$-equivariantly all over $p_{X}^{-1}(B)$, then the resulted $\widetilde{f} \mid p_{X}^{-1}(B)$ is transverse to $p_{Y}^{-1}(S)$ for each stratum $S$ of $Y$, because $p_{Y}^{-1}(S)$ is $\pi_{1}^{\text {orb }}(Y)$ invariant. Since there exists a countable family of such pairs $\left\{\left(B, B^{\prime}\right)\right\}$ with $\cup B^{\prime}=X_{0}$, we can $f_{*}$-equivariantly modify $\tilde{f}$ so that the new map $\tilde{f}^{\prime}$ is transverse over $p_{X}^{-1}\left(X_{0}\right)$. $\tilde{f}^{\prime}$ induces a map between $X$ and $Y$, which we denote by $f^{\prime}$. If the modification is sufficiently small, $f^{\prime}$ is still an orbi-map. In fact, choose a bouquet $C$ of finitely many circles in $X_{0}-f^{-1}(\Sigma Y)$ so that the inclusion map $C \rightarrow X_{0}-f^{-1}(\Sigma Y)$ induces an epimorphism on $\pi_{1}$. Choose $\tilde{f}^{\prime}$ sufficiently close to $\tilde{f}$ so that $f^{\prime}$ maps $C$ in $Y_{0}$, then $\pi_{1}\left(X_{0}-f^{\prime-1}(\Sigma Y)\right)$ maps onto $\pi_{1}^{\text {orb }}(X)$, and $f^{\prime}$ is an orbi-map.

Proof of Theorem 2.5. Without loss of generality we may assume that $f$ and $g$ are 0 -transverse. Then we can triangulate $X$ so that the 1 -skeleton of $X_{0}^{*}$ does not meet $f^{-1}(\Sigma Y) \cup g^{-1}(\Sigma Y)$. (See the proof of 2.4 for the notation.) We may further assume that $f$ and $g$ agree on the vertices of $X_{0}^{*}$. Let $\left(X_{0}^{*}\right)^{(i)}$ denote the $i$-skeleton of $X_{0}^{*}$. On $\left(X_{0}^{*}\right)^{(0)} \times I$, we use the constant homotopy. It extends over $\left(X_{0}^{*}\right)^{(1)} \times I$, because a loop in $Y_{0}$ bounds a disk in $Y$ if and only 
if it represents 1 in $\pi_{1}^{\text {orb }}(Y)$. Now we can use the asphericity of $Y$ to construct a desired orbi-homotopy. The argument is similar to the proof of 2.4 .

In general the composition of two orbi-maps may not be an orbi-map. But the following is obvious from the definition.

Proposition 2.10. Let $Y$ and $Z$ be good branchfolds, and let $f: X \rightarrow Y$ and $g: Y \rightarrow Z$ be 0-transverse orbi-maps. Then $g \circ f: X \rightarrow Z$ is an orbi-map and $(g \circ f)_{*}=g_{*} \circ f_{*}: \pi_{1}^{\text {orb }}(X) \rightarrow \pi_{1}^{\text {orb }}(Z)$.

Definition 2.11. An orbi-map $f: X \rightarrow Y$ is an orbi-homotopy equivalence if there exists an orbi-map $g: Y \rightarrow X$ such that $g \circ f$ and $f \circ g$ are orbihomotopic to the identity maps on $X$ and $Y$ respectively; $X$ and $Y$ are said to be orbi-homotopy equivalent.

An orbi-homotopy equivalence induces an isomorphism of orbifold fundamental groups. Conversely, combining 2.4, 2.5, 2.9, and 2.10, one deduces the following:

Corollary 2.12. Suppose $X$ and $Y$ are good compact aspherical branchfolds with isomorphic orbifold fundamental groups $\pi_{1}^{\text {orb }}(X) \cong \pi_{1}^{\text {orb }}(Y)$, then $X$ and $Y$ are orbi-homotopy equivalent. If we identify $\pi_{1}^{\mathrm{orb}}(X)$ and $\pi_{1}^{\mathrm{orb}}(Y)$ and denote the group by $G$, then $\tilde{X}$ and $\tilde{Y}$ are G-homotopy equivalent.

\section{TAME MAPS AND OR-MAPS}

In this section we consider a smaller class of maps. The notation in $\S 2$ will still be used. $X$ (resp. $Y$ ) will denote a connected $n$-dimensional (resp. $m$ dimensional) branchfold (possibly with boundary).

Definition 3.1. A map $f: X \rightarrow Y$ is tame if $f$ is an orbi-map satisfying $f\left(X_{0}\right) \subset Y_{0}$.

The following is immediate from a work of Fox [4] and the description of the universal covering orbifold and the orbifold fundamental group in $\S 2$.

Proposition 3.2. A continuous map $f: X \rightarrow Y$ is tame if and only if (1) $f\left(X_{0}\right) \subset$ $Y_{0}$, and (2) $f_{\sharp}\left(N_{X}\right) \subset N_{Y}$. Here $f_{\sharp}$ denotes the homomorphism $\pi_{1}\left(X_{0}\right) \rightarrow$ $\pi_{1}\left(Y_{0}\right)$ induced by $f$.

In [10] Takeuchi introduced the notion of OR-maps between branchfolds. A continuous map $f: X \rightarrow Y$ between branchfolds is an $O R$-map if the order of $\Gamma(f(x))$ divides the order of $\Gamma(x)$ for every $x \in X$.

Proposition 3.3 [10, p.483]. An OR-map $f: X \rightarrow Y$ is tame.

Proof. If $x \in X_{0}$, then $\Gamma(x)=1$ and hence $\Gamma(f(x))=1$. Therefore $f\left(X_{0}\right) \subset$ $Y_{0}$. Let $\mu_{i}$ be a meridian loop in $X_{0}$ about a codomension 2 stratum $S_{i} ; \mu_{i}$ is the boundary loop of a disk in $X$ which meets $\Sigma X$ transeversely at exactly one point, say $x$, of $S_{i}$. By continuity of $f$, we may assume that $f\left(\mu_{i}\right) \subset U_{f(x)}$, where $U_{f(x)}$ is a coordinate neighborhood of $f(x)$ in $Y$. The inclusion map 
$j: U_{f(x)} \rightarrow Y$ induces a map $j_{*}: \pi_{1}^{\text {orb }}\left(U_{f(x)}\right) \rightarrow \pi_{1}^{\text {orb }}(Y)$. The loop $f\left(\mu_{i}\right)$ represents an element of $\pi_{1}^{\text {orb }}\left(U_{f(x)}\right)$. Since $b_{i}(=$ the order of $\Gamma(x))$ is a multiple of the order of $\Gamma(f(x))=\pi_{1}^{\text {orb }}\left(U_{f(x)}\right), f\left(\mu_{i}^{b_{i}}\right)=f\left(\mu_{i}\right)^{b_{i}}$ represents 1 in $\pi_{1}^{\text {orb }}\left(U_{f(x)}\right)$. Therefore it represents 1 also in $\pi_{1}^{\text {orb }}(Y)$; i.e., $f_{\sharp}\left(\mu_{i}^{b_{i}}\right) \in N_{Y}$. Thus $f$ is tame.

On the other hand, a tame map is not necessarily an OR-map. For example, let $S^{2}(p)$ denote a teardrop orbifold whose underlying space is the 2dimensional sphere with a single cone point of angle $2 \pi / p$ at the North Pole. Then, whatever integers $p$ and $q$ are, the identity map from $S^{2}(p)$ to $S^{2}(q)$ is tame, but it is an OR-map only if $q$ divides $p$.

In general a restriction of a tame map $f: X \rightarrow Y$ to open subsets of $X$ and $Y$ may not be tame, whereas OR-maps always restrict to OR-maps.

Definition 3.4. A map $f: X \rightarrow Y$ is locally tame if the restriction $f: U_{x} \rightarrow$ $U_{f(x)}$ is tame for every $x \in X$, where $U_{x}$ and $U_{f(x)}$ are coordinate neighborhoods of $x$ and $f(x)$ with $f\left(U_{x}\right) \subset U_{f(x)}$. (The definition does not depend on the choice of such coordinate neighborhoods.)

A locally tame map $f$ induces a homomorphism $f_{*}: \pi_{1}^{\text {orb }}\left(U_{x}\right) \rightarrow \pi_{1}^{\text {orb }}\left(U_{f(x)}\right)$. Since these groups are identified with the local groups $\Gamma(x)$ and $\Gamma(f(x))$ at $x$ and $f(x)$, we have a homomorphism $f_{*}: \Gamma(x) \rightarrow \Gamma(f(x))$, which is independent of the choice of coordinate neighborhoods. The following is easily proved.

Proposition 3.5. (1) An OR-map is locally tame.

(2) If $Y$ is good, a tame map $f: X \rightarrow Y$ is locally tame.

Proposition 3.6. Let $f: X \rightarrow Y$ be a tame, locally tame map between branchfolds. Suppose that $X$ is good and that $f_{*}: \pi_{1}^{\mathrm{orb}}(X) \rightarrow \pi_{1}^{\mathrm{orb}}(Y)$ is injective. Then $f_{*}: \Gamma(x) \rightarrow \Gamma(f(x))$ is injective for every $x \in X$. In particular, $f$ maps $\Sigma X$ into $\Sigma Y$.

Proof of Proposition 3.6. Consider the following commutative diagram:

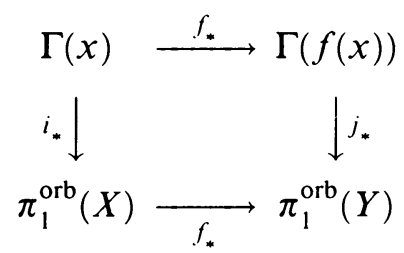

where the vertical arrows are induced from the inclusion maps $i: U_{x} \rightarrow X$ and $j: U_{f(x)} \rightarrow Y$. By assumption $i_{*}$ and the bottom $f_{*}$ are both injective; hence so is the top $f_{*}$.

Note that we still have the conclusion $f(\Sigma X) \subset \Sigma Y$ without the locallytameness condition on $f$. Choose a coordinate neighborhood $U_{x}$ about $x \in$ $\Sigma X$. There is a closed loop $\mu$ in $U_{x} \cap X_{0}$ which represents a nontrivial element in $\pi_{1}^{\text {orb }}\left(U_{x}\right)=\Gamma(x) ; \mu$ bounds a disk $D$ in $U_{x}$ such that $D \cap \Sigma X=\{x\}$. If 
$f(x) \notin \Sigma Y$, then $f(D) \subset Y_{0}$. Therefore $f_{*} \circ i_{*}(\mu)=1$ in $\pi_{1}^{\text {orb }}(Y)$, which contradicts the assumption that $f_{*}$ and $i_{*}$ are injective.

Corollary 3.7. Let $f: X \rightarrow Y$ be an OR-map between branchfolds. Suppose that $X$ is good and that $f_{*}: \pi_{1}^{\mathrm{orb}}(X) \rightarrow \pi_{1}^{\mathrm{orb}}(Y)$ is injective. Then $f_{*}: \Gamma(x) \rightarrow$ $\Gamma(f(x))$ is a group isomorphism.

Corollary 3.8. Let $X, Y$ and $f: X \rightarrow Y$ be as in 3.7. Then the following holds. (1) If $S$ is a stratum of $X$, then $f(S) \subset T$ for some stratum $T$ of $Y$. If $S^{\prime}(\neq S)$ is a stratum of $X$ contained in the closure of $S$, then $f\left(S^{\prime}\right) \subset T^{\prime}$ for some stratum $T^{\prime}$ of $Y$ with $T^{\prime} \neq T$.

(2) If $X$ and $Y$ are locally orientable orbifolds (i.e., the local groups are subgroups of $S O(n)), \operatorname{dim}(X) \leq 3$, and $\operatorname{dim}(Y) \leq 3$, then each stratum of $X$ is mapped into a stratum of $Y$ of the same codimension.

(3) If $f(X)$ meets every stratum of $Y$, then $Y$ is also good.

Proof. (1) and (3) are obvious. (2) holds, because in these dimensions faithful representations (in $S O(n)$ ) of a fixed finite group are linearly equivalent. See $[2, \S \S 2,3]$.

\section{T-HOMOTOPY EQUIVALENCES AND OR-HOMOTOPY EQUIVALENCES}

Definition 4.1. Let $X$ and $Y$ be branchfolds. A homotopy $\left\{f_{s}\right\}_{s \in I}: X \times I \rightarrow Y$ is called a t-homotopy if it is tame as a map (or, equivalently, if $f_{s}: X \rightarrow Y$ is tame for every $s \in I$ ), and $f_{0}$ and $f_{1}$ are said to be t-homotopic. (Note that if $f_{s}\left(X_{0}\right) \subset Y_{0}$ for every $s$ and $f_{0}$ is tame, then the homotopy $\left\{f_{s}\right\}$ is a t-homotopy.) A tame map is a t-homotopy equivalence if there exists a tame map $g: Y \rightarrow X$ (called a t-homotopy inverse) such that $g \circ f$ and $f \circ g$ are t-homotopic to the identity maps on $X$ and $Y$ respectively. $O R$ homotopy equivalences are defined similarly. A homeomorphism $f: X \rightarrow Y$ is a t-homeomorphism if $f$ and its inverse $f^{-1}$ are both tame.

If $f: X \rightarrow Y$ is a t-homeomorphism between good branchfolds $X$ and $Y$, then $f_{*}: \Gamma(x) \rightarrow \Gamma(f(x))$ is an isomorphism for every $x \in X$, and $f$ is a topological isomorphism of orbifolds. In particular the two representations $\Gamma(x)$ and $\Gamma(f(x))$ are topologically equivalent for every $x$. If the order is odd, these are linearly equivalent $[5,7]$.

The author does not know whether a good branchfold can be t-homeomorphic or even t-homotopy equivalent to a bad one or not. Below we will see that good 2-dimensional closed branchfolds are never t-homotopy equivalent to bad 2dimensional closed branchfolds. Also one deduces the following from 3.8(3).

Theorem 4.2. Suppose $X$ and $Y$ are OR-homotopy equivalent branchfolds. Then $X$ is good if and only if $Y$ is good.

It is well known that every homotopy equivalence between closed surfaces is homotopic to a homeomorphism. The following analogue holds true for branchfolds. 
Theorem 4.3. Any t-homotopy equivalence between closed 2-dimensional branchfolds is t-homotopic to a t-homeomorphism.

Corollary 4.4. Every OR-homotopy equivalence between closed 2-dimensional branchfolds is OR-homotopic to an isomorphism.

We use the following lemma to prove Theorem 4.3.

Lemma 4.5. Let $X$ be a 2-dimensional branchfold. If a tame map $f: X \rightarrow X$ is t-homotopic to the identity map on $X$, then $f(x)=x$ for every $x \in \Sigma X$.

Proof of Lemma 4.5. Let $\left\{f_{s}: X \rightarrow X\right\}$ be a t-homotopy between $f_{0}=1_{X}$ and $f_{1}=f$, and let $x$ be a point in $\Sigma X$. The set $J=\left\{t \in I \mid f_{s}(x)=x\right.$ for every $s \in$ $[0, t]\}$ is a closed subset of $I$, because $f_{s}(x)$ is continuous with respect to $s$. $J$ is not empty, because $0 \in J$. So $J$ has a maximum $\tau$. Let $U_{x}$ be a coordinate neighborhood of $x$. The interval $[0, \tau]$ is compact, so there exists a closed disk neighborhood $D$ of $x$ in $X$ such that $f_{s}(D) \subset U_{x}$ for every $s$ in $[0, \tau]$. As $f_{s}(D-\{x\}) \subset U_{x}-\{x\}, f_{s}(\partial D)$ represents the generator of $\pi_{1}\left(U_{x}-\{x\}\right)=\mathbf{Z}$ and $f_{s}(D)$ is a neighborhood of $x$ for every $s \in[0, \tau]$. In particular $f_{\tau}(D)$ is a neighborhood of $x$. Suppose $\tau<1$. If $\epsilon(>0)$ is sufficiently small, $f_{\tau+\epsilon}(D)$ is still a neighborhood of $x$, and one deduces that $f_{\tau+\epsilon}(x)=x$. This contradicts the maximality of $\tau$. Therefore $\tau=1$ and the lemma is proved.

Proof of Theorem 4.3. Let $f: X \rightarrow Y$ be a thomotopy equivalence between branchfolds. Take a point $x$ of $\Sigma X$, then the lemma above applied to $g \circ f$ implies that $g \circ f(x)=x$. As $g$ is tame, $f(x) \in \Sigma Y$. Similarly $f \circ g(y)=y$ and $g(y) \in \Sigma X$ for every $y \in \Sigma Y$. Thus $f$ defines a bijection between $\Sigma X$ and $\Sigma Y$. If $\Sigma X$ is empty, then $\Sigma Y$ is also empty and the result is well known as mentioned above. So assume that $\Sigma X$ is not empty. For every $x \in \Sigma X$, we may assume that $f$ is a homeomorphism when restricted to small closed disk neighborhoods $D_{x}$ and $D_{f(x)}$ of $x$ and $f(x)$, by 4.5. We may further assume that $f$ sends $M=X-\bigcup_{x \in \Sigma X} \operatorname{int}\left(D_{x}\right)$ into $N=Y-\bigcup_{y \in \Sigma Y} \operatorname{int}\left(D_{y}\right)$, where $\operatorname{int}(-)$ denotes the interior. The restriction $f_{0}: M \rightarrow N$ is a homotopy equivalence and is a homeomorphism $\partial M \rightarrow \partial N$ on boundary. Therefore $f_{0}$ is homotopic rel $\partial$ to a homeomorphism, and hence $f$ is t-homotopic to a tame homeomorphism $h$. Its inverse $h^{-1}$ sends $Y_{0}$ into $X_{0}$. Note that $h \circ g$ is t-homotopic to $1_{Y}$. Therefore $h^{-1}$ is homotopic rel $\Sigma Y$ to $g$ through a homotopy which sends $Y_{0}$ into $X_{0}$. As remarked in 4.1, this implies that $h^{-1}$ is tame.

\section{FinAL REMARKS}

It is important to decide when an orbi-homotopy equivalence is orbi-homotopic to an isomorphism of orbifolds. One way to attack this problem is to use equivariant simple-homotopy theory and equivariant surgery theory as was 
done in $[1,3]$. Another possible way is to develop simple-homotopy theory and surgery theory directly for orbifolds and orbi-maps.

There is an obvious obstruction to deforming an orbi-homotopy eauivalence $f: X \rightarrow Y$ into an isomorphism. Since an isomorphism is a tame map, the composition

$$
f_{*} \circ q_{X}: \pi_{1}\left(X_{0}\right) \longrightarrow \pi_{1}^{\mathrm{orb}}(X) \longrightarrow \pi_{1}^{\mathrm{orb}}(Y)
$$

must lift to a homomorphism $\pi_{1}\left(X_{0}\right) \rightarrow \pi_{1}\left(Y_{0}\right)$, and there is a group theoretic obstruction to this defined in $H^{2}\left(\pi_{1}\left(X_{0}\right), N_{Y} /\left[N_{Y}, N_{Y}\right]\right)$, where $\left[N_{Y}, N_{Y}\right]$ is the commutator subgroup of $N_{Y}$.

\section{REFERENCES}

1. F. Connolly and T. Kozniewski, Classification of crystallographic manifolds with odd order holonomy, (preprint).

2. M. W. Davis and J. W. Morgan, Finite group actions on homotopy 3-spheres, The Smith Conjecture, Pure and Appl. Math. 112 (Bass and Morgan, eds.), Academic Press, Orlando, Florida, 1984, pp. 181-225.

3. S. Ferry, J. Rosenberg and S. Weinberger, Equivariant topological rigidity phenomena, C. R. Acad. Sci. Paris 306 I (1988), 777-782.

4. R. H. Fox, Covering spaces with singularities, Algebraic Geometry and Topology, Princeton Univ. Press, Princeton, New Jersey, 1957, pp. 243-257.

5. W. C. Hsiang and W. Pardon, When are topologically equivalent orthogonal representations equivalent?, Invent. Math. 68 (1982), 275-316.

6. M. Kato, On uniformizations of orbifolds, Homotopy Theory and Related Topics, Adv. Studies in Pure Math. 9 (H. Toda, ed.), Kinokuniya, Tokyo and North-Holland, Amsterdam, 1986, pp. 149-172.

7. I. Madsen and M. Rothenberg, Classifying G-spheres, Bull. Amer. Math. Soc. 7 (1982), 223-226.

8. W. H. Meeks, III and S.-T. Yau, Group actions on $\mathbf{R}^{3}$, The Smith Conjecture, Pure and Appl. Math. 112 (Bass and Morgan, eds.), Academic Press, Orlando, Florida, 1984, pp. 167-179.

9. P. Scott, The geometries of 3-manifolds, Bull. London Math. Soc. 15 (1983), 401-487.

10. Y. Takeuchi, A clssification of a class of 3-branchfolds, Trans. Amer. Math. Soc. 307 (1988), 481-502.

11. Waldhausen's classification theorem for finitely uniformizable 3-orbifolds, (preprint).

12. W. Thurston, The geometry and topology of three manifolds, Lecture Notes, Dept. of Math., Princeton Univ., Princeton, NJ, (1976-1979).

Department of Mathematics, Josai University, Sakado, Saitama, Japan 